\title{
Rare Primary Pulmonary Sarcomatoid Carcinoma with Isolated Pancreas Metastasis: Case Report and Literature Review
}

\author{
Nie Zhang1\#, Hui Qiu ${ }^{2,3 \#, ~ Z i q i n ~ C h e n ~}{ }^{1}$, Wanming Liu ${ }^{1}$, Xueting Chen ${ }^{1}$, Tingting Wang ${ }^{1}$, Zhengqiu \\ $\mathrm{Zhu}^{4 *}$ and Longzhen Zhang ${ }^{2,3 *}$ \\ ${ }^{1}$ First Clinical College, Xuzhou Medical University, China \\ ${ }^{2}$ Cancer Institute, Xuzhou Medical University, China \\ ${ }^{3}$ Department of Radiation Oncology, Affiliated Hospital of Xuzhou Medical University, China \\ ${ }^{4}$ Department of Medical Oncology, Affiliated Hospital of Xuzhou Medical University, China \\ \#These authors have contributed equally to this work and share first authorship. \\ *Corresponding author: Longzhen Zhang, Cancer Institute, Xuzhou Medical University, Xuzhou, Jiangsu, China and Zhengqiu Zhu, \\ Department of Medical Oncology, Affiliated Hospital of Xuzhou Medical University, Xuzhou, Jiangsu, China
}

\section{ARTICLE INFO}

Received: 幽 December 15, 2021

Published: 蔧 January 05, 2022

Citation: Nie Zhang, Hui Qiu, Ziqin Chen, Zhengqiu Zhu, Longzhen Zhang, et al., Rare Primary Pulmonary Sarcomatoid Carcinoma with Isolated Pancreas Metastasis: Case Report and Literature Review. Biomed J Sci \& Tech Res 40(5)2022. BJSTR. MS.ID.006511.

Keywords: Pulmonary Sarcomatoid Carcinoma; Pancreas Metastasis; Rare Malignancy; Da Vinci Surgery; Case Report

\begin{abstract}
Pulmonary sarcomatoid carcinoma (PSC) is a very rare subtype of non-small cell lung cancer (NSCLC) and has an extremely poor prognosis with 5-year overall survival rate ranging from $10 \%$ to $21 \%$, and it accounts for approximately $0.4 \%$ of all pulmonary malignancies. Compared with other types of NSCLC, PSC is more prone to invasion and distant metastasis, and the most common metastatic sites are bone, adrenal gland and brain. The incidence of pancreatic metastasis of malignant tumors is uncommon clinically, and the occurrence of PSC with pancreatic metastasis is even rarer. Here, we describe a case of PSC with isolated pancreas metastasis that was initially considered as acute pancreatitis. After multidisciplinary discussion, the patient underwent stateof-the-art Da Vinci robotic surgery and simultaneous resection of the pancreatic and pulmonary masses followed by multiple cycles of chemotherapy. Soon after, the patient progressed and the treatment was changed to immunotherapy combined with antiangiogenesis therapy. Since the patient developed an acute episode of immunological enteritis, the therapeutic schedule was adjusted to maintenance therapy with Anlotinib subsequently, and we now estimate that the patient's subsequent survival time is relatively short.
\end{abstract}

Abbreviations: PSC: Pulmonary Sarcomatoid Carcinoma; NSCLC: Non-Small Cell Lung Cancer; CT: Computed Tomography; PET: Positron Emission Tomography

\section{Introduction}

Sarcomatoid change is an uncommon phenomenon (accounts for approximately $0.4 \%$ ) in non-small cell lung cancer (NSCLC) which is characterized by poorly differentiated neoplasm containing sarcoma or sarcomatoid components [1-3], its pathological diagnosis include five subtypes (pleomorphic carcinoma, spindle cell carcinoma, carcinosarcoma, giant cell carcinoma and pulmonary blastom) and require high-quality sampling of the tumor [4]. Since pulmonary sarcomatoid carcinoma (PSC) is generally a form of highly aggressive malignancy in NSCLC, it is no wonder that it has an extremely poor prognosis [1]. Previous studies have shown 
that patients with PSC respond poorly to platinum based standard regimens and develop easily to chemotherapeutic resistance [2,5], and thus it is absolutely essential to explore and work out a more effective therapeutic strategy. Here, we reported a 59-year-old patient who was admitted for attacks of acute pancreatitis and was diagnosed with PSC with isolated pancreas metastasis, an even rarer malignance. Then, we reviewed the literature available and hope to seek out safer and more effective treatment strategies which can improve the prognosis of PSC patients.

\section{Case Description}

A 59-year-old male, an active smoker with a 40-year history of tobacco smoking (an average of 40 cigarettes per day), presented to a hospital in Xuzhou on November 26, 2020, he complained of persistent epigastric pain for half a day after heavy drinking. The patient denied any previous history of respiratory or digestive disease and malignancies. The results of hematological examination showed a mild leukocytosis and hyperamylasemia (130U/L; reference range: $30-110 \mathrm{U} / \mathrm{L}$ ), therefore, the patient was initially thought to had acute pancreatitis. However, According to the images of chest and abdomen computed tomography (CT), a lesion $(46 \mathrm{~mm} \times 31 \mathrm{~mm})$ in the right lower lung lobe was detected; a cystic lesion was existed in the body of pancreas, and cystadenocarcinoma remained to be ruled out. In order to further clarify the patient's condition and diagnosis, the patient then underwent positron emission tomography (PET)/CT. As shown in Figure 1, the two nodules which located in the inferior lobe of right lung and the body of pancreas had significantly increased fluorodeoxyglucose metabolism and were considered to be malignant lesions. Then, the patient underwent the CT-guided percutaneous pulmonary nodules puncture biopsy, and the pathological diagnosis was poorly differentiated lung adenocarcinoma.
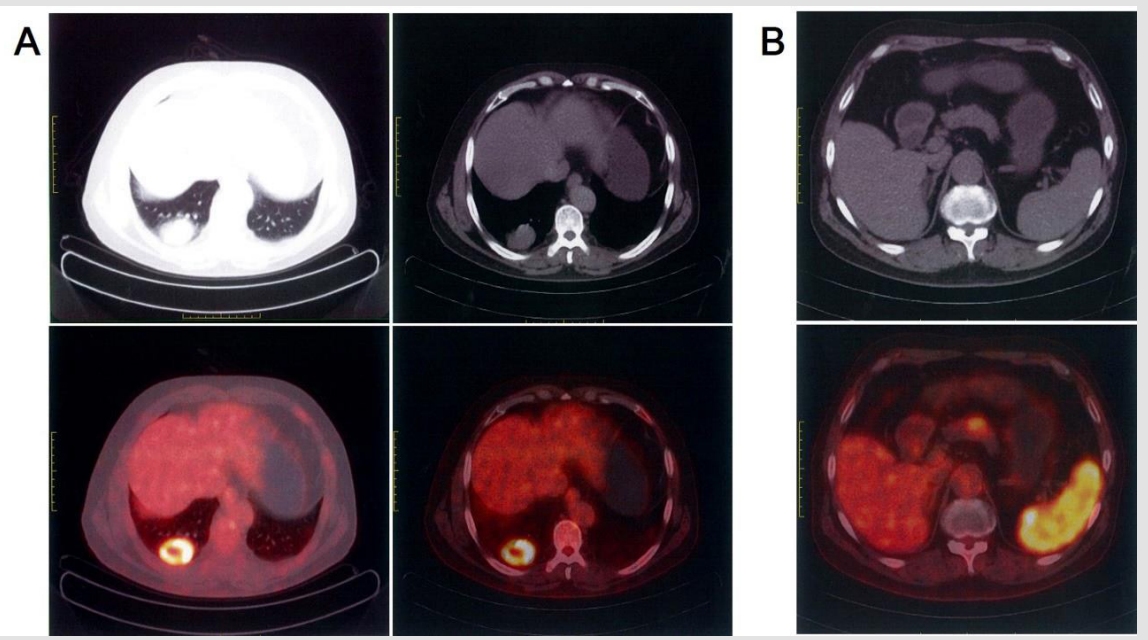

Figure 1: Partial PET/CT scanned images of the patient's chest and abdomen. The nidus in the inferior lobe of right lung (A) and the body of pancreas (B) had significantly increased fluorodeoxyglucose metabolism and were considered to be malignant lesions.

In order to receive further comprehensive treatments, the patient was subsequently admitted to the Affiliated Hospital of Xuzhou Medical University. The result of magnetic resonance cholangiopancreatography showed a $1.1 \mathrm{~cm} \times 1.5 \mathrm{~cm}$ lesion in the pancreatic body (Figure 2). Considering that the lesions on the lung and pancreas were isolated and the patient was in good general condition with acceptable examination results of blood routine, liver and kidney function, serum electrolytes and coagulation function, the patient underwent the state-of-the-art Da Vinci robotic assisted laparoscopic resection of pancreatic lesions, thoracoscopic lobectomy and intrathoracic lymph node dissection on December
15, 2020 after excluding the surgical contraindications and thorough communication with the patient and his families, and the patient recovered well after surgery. The postoperative pathological results demonstrated that the two lesions in the inferior lobe of right lung and the body of pancreas were malignant with extensive necrosis, and the tumor sizes were $1.5 \mathrm{~cm} \times 1.5 \mathrm{~cm} \times 0.5 \mathrm{~cm}$ (pulmonary lesion) and $1.3 \mathrm{~cm} \times 1.1 \mathrm{~cm} \times 1 \mathrm{~cm}$ (pancreatic lesion), respectively, no definite vascular and nerve invasion, no cancer cell involvement at the bronchial cut-off, vascular cut-off and the cross-section of pancreas, no tumor metastasis was detected in the group 7 and 11 lymph nodes. 

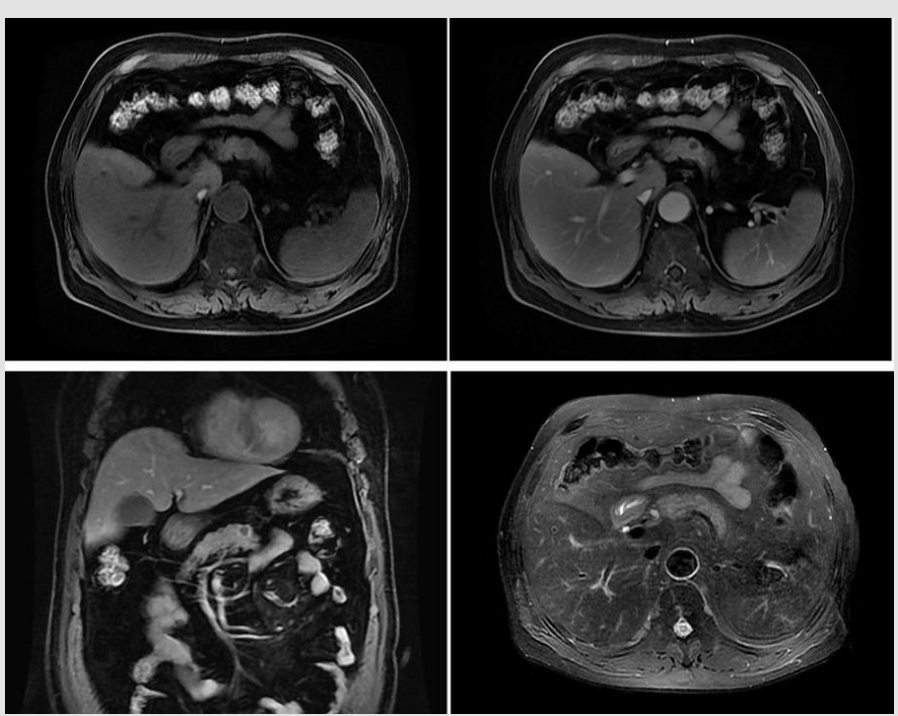

Figure 2: Representative images of magnetic resonance cholangiopancreatography of the patient. The examination revealed a $1.1 \mathrm{~cm} \times 1.5 \mathrm{~cm}$ lesion in the pancreatic body.

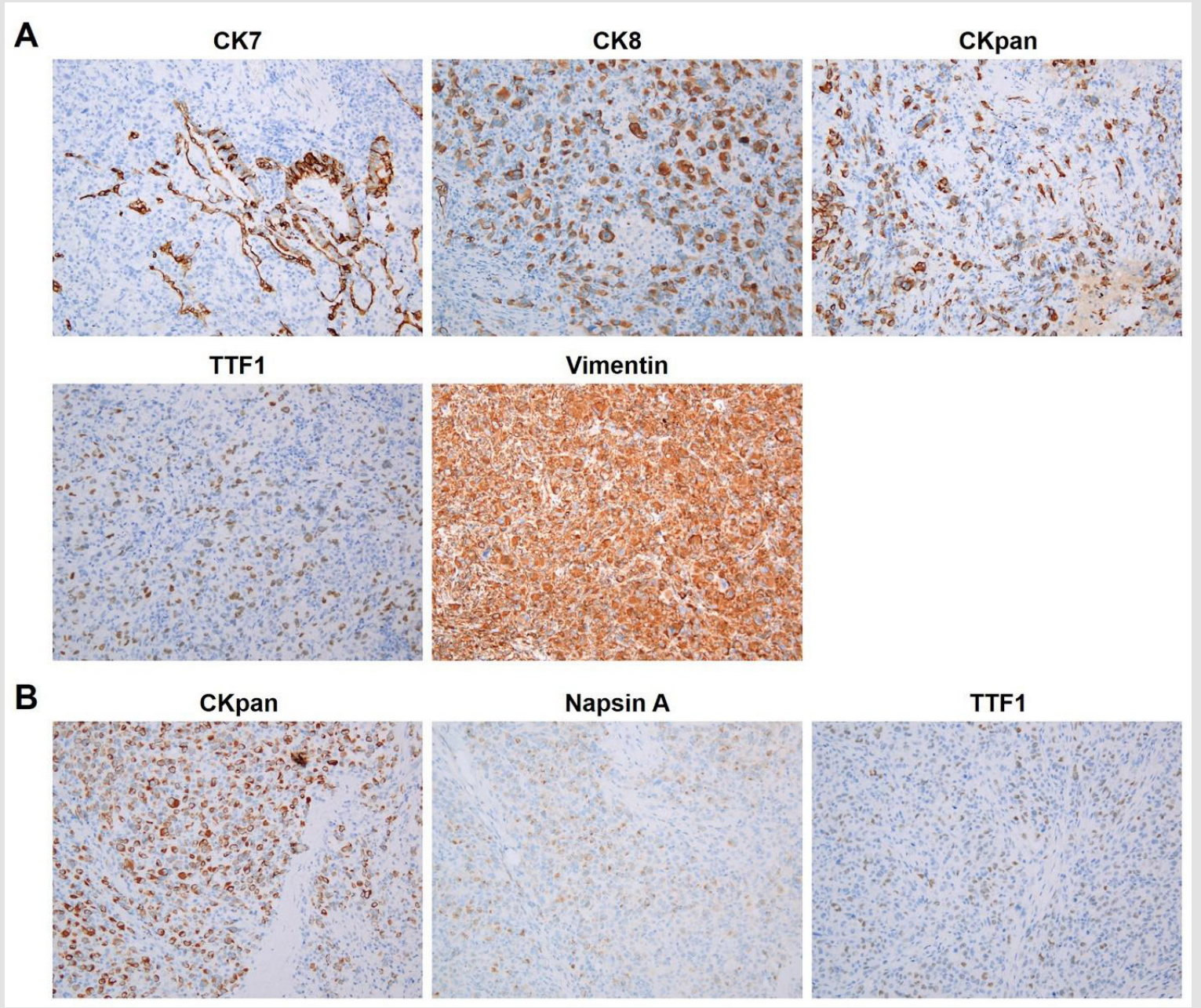

Figure 3:

A. Immunohistochemical staining results of CK7, CK8, CKpan, TTF1, Vimentin in lung tumor tissue and

B. CKpan, NapsinA, TTF-1 in pancreatic metastasis tissue. 
The immunohistochemistry (IHC) results of lung tumor (Figure 3A) were Ki67 (+, about 50\%), TTF-1 (partial +), CD56 (-), Syn $(-)$, CgA (-), P40 (-), CK5/6 (-), CK7 (focal +), NapsinA (partial $+)$, CKpan (+), CK8/18 (+), Vimentin (+), S100 ( \pm ), HMB45 (-), Melan-A (focal +), LCA (CD45)(-), ALK (-), CD30 (-), DPC4 (+), Bcl-10 (-), PAS (several endochylema + ), which corresponded to poorly differentiated carcinoma with a tendency to sarcomatoid carcinoma. The pancreatic tumor was consider metastatic poorly differentiated carcinoma with TTF-1 (partial +), NapsinA (+), CKpan $(+)$ and Bcl-10 (-) (Figure 3B). Overall, the patient was definitively diagnosed with the right PSC with isolated pancreatic metastasis. According to the 2020 National Comprehensive Cancer Network guideline, the patient received the first cycle of chemotherapy with Gemcitabine (2g, day1 and 7) combined with Carboplatin $(800 \mathrm{mg}$, day 1) on January 20, 2021. However, the patient's platelet level significantly decreased and the lowest number was $30^{*} 109 / \mathrm{L}$ on the $13^{\text {th }}$ day after chemotherapy, hence, the chemotherapy regimen was adjusted to Abraxane (400mg, day1) combined with Nedaplatin (40mg, days 2 to 3), and the patient regularly received four cycles without severe adverse reactions.

Shortly thereafter, the patient developed tumor recurrence and metastasis which were indicated by the CT examination on June 22 ,
2021, a new mass with uneven enhancement appeared in the upper lobe of right lung, suggesting tumor metastasis; multiple nodular high-density shadows appeared in the liver and stomach space which were considered as lymph node metastasis. Due to the high expression level of PD-L1 (+, tumor proportion score was about 70\%) in the patient's tumor tissue, the patient's therapeutic regimen was then adjusted to Camrelizumab (200mg) combined with Anlotinib (12mg per day, days 1to 14, 21 days per cycle). However, the patient presented with abdominal pain with hematochezia on the 15th day after the immunotherapy combined with targeted therapy. After comprehensive examinations, the patient was considered to have an acute episode of immunological enteritis, and the new nidus in the right adrenal area was considered metastatic. The patient subsequently received comprehensive treatments of antiinflammatory, analgesic, fluid replacement and electrolyte balance, the symptoms were gradually controlled and relieved. Considering the recent significant weight loss (Figure 4A) and poor appetite of the patient, it was not suitable for chemotherapy. Therefore, the patient is now receiving maintenance therapy with Anlotinib. The patient's therapeutic process has been summarized as shown in Figure 4B, and we now estimate that the patient's subsequent survival time is relatively short.
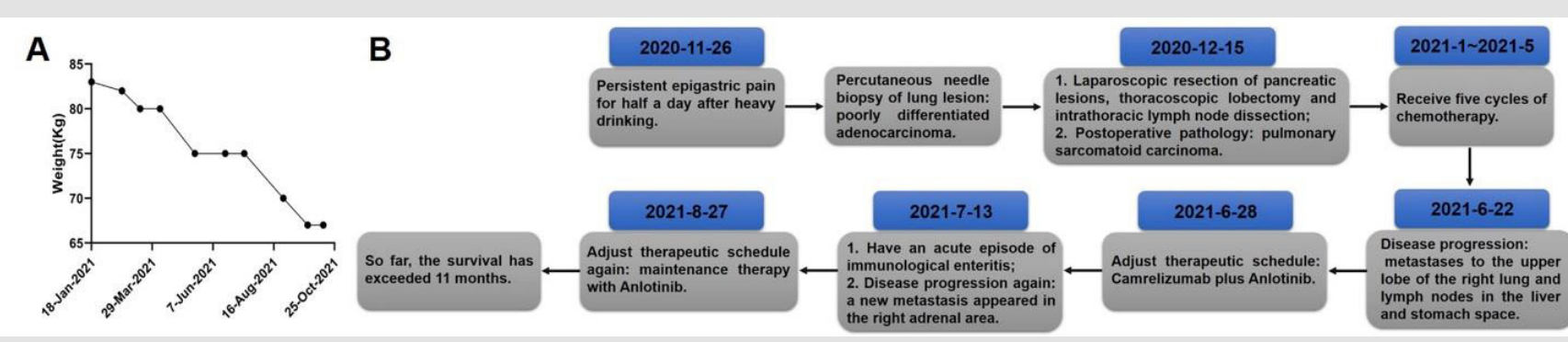

Figure 4:

A. Weight change curve of the patient during treatment and

B. The summary of his therapeutic process.

\section{Discussion}

Pancreatic metastasis of lung cancer is rare, its frequency was mainly related to the histological types and small cell lung carcinoma has the highest incidence which was followed by lung adenocarcinoma [6], while the morbidity of PSC with pancreatic metastasis was extremely low. In this study, we reported a patient who suffered from PSC with isolated pancreas metastasis, after simultaneous resection of the pulmonary and pancreatic masses by state-of-the-art Da Vinci robotic surgery, the patient underwent multiple cycles of chemotherapy, immunotherapy and antiangiogenesis therapy, and he has survived for more than 11 months.

Due to the highly malignant and aggressive characteristics of this tumor, the vast majority of patients with PSC are tended to presented at advanced stage at the time of diagnosis, thus losing the opportunity of surgical resection, therefore, effective systemic treatments are of great importance. It is widely known that the determination of effective therapeutic strategies should be based on well-defined molecular mechanisms of tumorigenesis and cancer progression, whereas little was known about PSC's molecular pathogenesis now because of its rarity and heterogeneous morphology [2].

In order to describe the mutational profile of sarcomatoid carcinoma, Fallet V, et al. used high-throughput genotyping technology to test 114 surgical biopsies from 81 patients with sarcomatoid carcinoma for 214 mutations affecting 26 oncogenes and tumor suppressor genes, they found that the most frequent mutations were KRAS, EGFR, TP53, STK11, NOTCH1, NRAS and PI3KCA, and the EGFR mutations were almost always rare 
mutations (89\%) [7], and this result had been confirmed by Terra $\mathrm{SB}$, et al. [8]. The above finding suggested that testing for targetable mutations should be considered for patients with PSC, because a subpopulation may benefit from some molecular targeted antitumor drugs which have been approved for clinical use. Another promising new therapeutic strategy of PSC is the use of immune checkpoint inhibitors, because several studies had illustrated that PD-L1 was overexpressed in PSC [9-11]. Moreover, Pecuchet N, et al. demonstrated that, compared with the PSCs with APOBEC enzyme and homologous recombination deficiency, the PSC patients with tobacco-associated signatures had a higher rate of PD-L1 overexpression and a poorer overall survival [12]. The growing body of studies regarding the molecular mechanisms of PSC support that the malignant tumor is not only morphologically diverse, but also quite genetically diverse. Hence, the discovery and description of genetic events might provide novel treatment choices for PSCs, and potentially improve the patients' prognosis and bring PSC into the era of targeted therapy and immunotherapy.

\section{Conclusion}

In this study, we described an extremely rare case of PSC with isolated pancreas metastasis which was presented as acute pancreatitis initially. For PSC patients with isolated metastasis, if the patient's physical condition permit, surgical resection of the lesions could be considered and thus obtain high-quality tissue specimens for gene detection, which would provide a stronger basis for the selection of subsequent systemic therapy strategies including gene targeting treatment and immunotherapy.

\section{Data Availability Statement}

The original contributions presented in the study are included in the article. Further inquiries can be directed to the corresponding authors.

\section{Ethics Statement}

The study involving human participant was reviewed and approved by the ethics committee of the Affiliated Hospital of Xuzhou Medical University. The patient provided his written informed consent to participate in this study.

\section{Conflict of Interest}

The authors declare that the study was conducted in the absence of any commercial or financial relationships that could be construed as a potential conflict of interest.

\section{Author Contributions}

LZ, ZZ and NZ contributed to the conception, design and supervision of this study; NZ and HQ drafted and revised the manuscript; NZ, HQ ZQ and WM were involved directly or indirectly in the care of the patient; NZ, HQ, XT and TT participated in the collection of case data. All authors contributed to the article and approved the submitted version.

\section{Funding}

This research was funded by National Natural Science Foundation of China, grant number 81972845; Introduction of Specialist Team in Clinical Medicine of Xuzhou, grant number 2019TD003; Science and Technology Project of Xuzhou, grant number KC21168.

\section{Acknowledgment}

Not applicable.

\section{References}

1. Yendamuri S, Caty L, Pine M, Adem S, Bogner P, et al. (2012) Outcomes of sarcomatoid carcinoma of the lung: a Surveillance, Epidemiology, and End Results Database analysis. Surgery 152(3): 397-402.

2. Boland JM, Mansfield AS, Roden AC (2017) Pulmonary sarcomatoid carcinoma-a new hope. Ann Oncol 28(7): 1417-1418.

3. Domblides C, Leroy K, Monnet I, Mazieres J, Barlesi F, et al. (2020) Efficacy of Immune Checkpoint Inhibitors in Lung Sarcomatoid Carcinoma. J Thorac Oncol 15(5): 860-866.

4. Smadhi H, Boudaya MS, Abdannadher M, Ben Abdelghaffar H, Kamoun $\mathrm{H}$, et al. (2019) Pulmonary Sarcomatoid carcinoma: a surgical diagnosis and prognostic factors. Tunis Med 97(1): 128-132.

5. Vieira T, Girard N, Ung M, Monnet I, Cazes A, et al. (2013) Efficacy of first-line chemotherapy in patients with advanced lung sarcomatoid carcinoma. J Thorac Oncol 8(12): 1574-1577.

6. Liratzopoulos N, Efremidou EI, Papageorgiou MS, Romanidis K, Minopoulos GJ, et al. (2006) Extrahepatic biliary obstruction due to a solitary pancreatic metastasis of squamous cell lung carcinoma. Case report. J Gastrointestin Liver Dis 15(1): 73-75.

7. Fallet V, Saffroy R, Girard N, Mazieres J, Lantuejoul S, et al. (2015) Highthroughput somatic mutation profiling in pulmonary sarcomatoid carcinomas using the LungCarta Panel: exploring therapeutic targets. Ann Oncol 26(8): 1748-1753.

8. Terra SB, Jang JS, Bi L, Kipp BR, Jen J, et al. (2016) Molecular characterization of pulmonary sarcomatoid carcinoma: analysis of 33 cases. Mod Pathol 29(8): 824-831.

9. Velcheti V, Rimm DL, Schalper KA (2013) Sarcomatoid lung carcinomas show high levels of programmed death ligand-1 (PD-L1). J Thorac Oncol 8(6): 803-805.

10. Kim S, Kim MY, Koh J, Go H, Lee DS, et al. (2015) Programmed death-1 ligand 1 and 2 are highly expressed in pleomorphic carcinomas of the lung: Comparison of sarcomatous and carcinomatous areas. Eur J Cancer 51(17): 2698-2707.

11. Vieira T, Antoine M, Hamard C, Fallet V, Duruisseaux M, et al. (2016) Sarcomatoid lung carcinomas show high levels of programmed death ligand-1 (PD-L1) and strong immune-cell infiltration by TCD3 cells and macrophages. Lung Cancer 98: 51-58.

12. Pecuchet N, Vieira T, Rabbe N, Antoine M, Blons H, et al. (2017) Molecular classification of pulmonary sarcomatoid carcinomas suggests new therapeutic opportunities. Ann Oncol 28(7): 1597-1604. 
ISSN: 2574-1241

DOI: 10.26717/BJSTR.2022.40.006511

Zhengqiu Zhu, Longzhen Zhang. Biomed J Sci \& Tech Res

(C) (P) This work is licensed under Creative

Submission Link: https://biomedres.us/submit-manuscript.php

\begin{tabular}{ll} 
BIOMEDICAL & Assets of Publishing with us \\
RESEARCHES & - Global archiving of articles \\
\hline I Immediate, unrestricted online access
\end{tabular}

